\title{
Neurological Complications of Lyme Disease
}

National Institute of Neurological Disorders and Stroke (NINDS)

\section{Source}

National Institute of Neurological Disorders and Stroke (NINDS). Neurological

Complications of Lyme Disease Information Page.

Lyme disease is caused by a bacterial organism that is transmitted to humans via the bite of an infected tick. Most people with Lyme disease develop a characteristic skin rash around the area of the bite. The rash may feel hot to the touch, and vary in size, shape, and color, but it will often have a "bull's eye" appearance (a red ring with a clear center). However, there are those who will not develop the rash, which can make Lyme disease hard to diagnose because its symptoms and signs mimic those of many other diseases.

Anywhere from 7 to 14 days (or in some cases, 30 days) following an infected tick's bite, the first stage of Lyme disease may begin with flu-like symptoms such as fever, chills, swollen lymph nodes, headaches, fatigue, muscle aches, and joint pain.

Neurological complications most often occur in the second stage of Lyme disease, with numbness, pain, weakness, Bell's palsy (paralysis of the facial muscles), visual disturbances, and mening itis symptoms such as fever, stiff neck, and severe headache. Other problems, which may not appear until weeks, months, or years after a tick bite, include decreased concentration, irritability, memory and sleep disorders, and nerve damage in the arms and legs. 